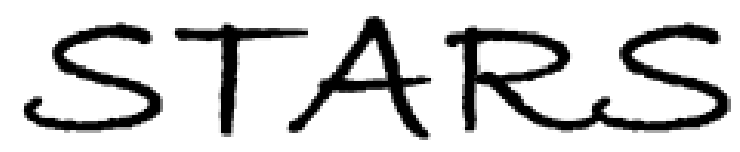

University of Central Florida

STARS

$1-1-2009$

\title{
Spitzer Observations of Comet 67p/Churyumov-Gerasimenko At 5.5-4.3 Au From The Sun
}

\author{
Michael S. Kelley \\ University of Central Florida \\ Diane $\mathrm{H}$. Wooden \\ Cecilia Tubiana \\ Hermann Boehnhardt \\ Charles E. Woodward
}

See next page for additional authors

Find similar works at: https://stars.library.ucf.edu/facultybib2000

University of Central Florida Libraries http://library.ucf.edu

This Article is brought to you for free and open access by the Faculty Bibliography at STARS. It has been accepted for inclusion in Faculty Bibliography 2000s by an authorized administrator of STARS. For more information, please contactSTARS@ucf.edu.

\section{Recommended Citation}

Kelley, Michael S.; Wooden, Diane H.; Tubiana, Cecilia; Boehnhardt, Hermann; Woodward, Charles E.; and Harker, David E., "Spitzer Observations of Comet 67p/Churyumov-Gerasimenko At 5.5-4.3 Au From The Sun" (2009). Faculty Bibliography 2000s. 1713.

https://stars.library.ucf.edu/facultybib2000/1713

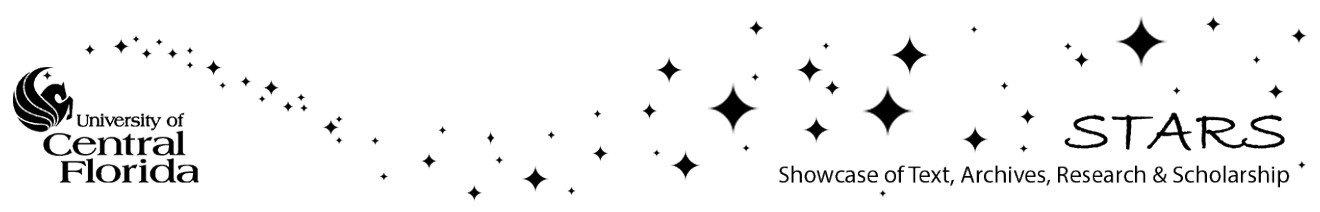


Authors

Michael S. Kelley, Diane H. Wooden, Cecilia Tubiana, Hermann Boehnhardt, Charles E. Woodward, and David E. Harker 


\title{
SPITZER OBSERVATIONS OF COMET 67P/CHURYUMOV-GERASIMENKO AT 5.5-4.3 AU FROM THE SUN
}

\author{
Michael S. Kelley ${ }^{1,6}$, Diane H. Wooden ${ }^{2}$, Cecilia Tubiana ${ }^{3}$, Hermann Boehnhardt ${ }^{3}$, Charles E. Woodward ${ }^{4}$, \\ AND DAVID E. HARKER ${ }^{5}$ \\ ${ }^{1}$ Department of Physics, University of Central Florida, 4000 Central Florida Blvd., Orlando, FL 32816-2385, USA \\ ${ }^{2}$ NASA Ames Research Center, Space Science Division, MS 245-3, Moffett Field, CA 94035, USA; Diane.H.Wooden@ nasa.gov \\ ${ }^{3}$ Max Planck Institute for Solar System Research, Max-Planck-Str. 2, 37191 Katlenburg-Lindau, Germany; boehnhardt@mps.mpg.de, tubiana@mps.mpg.de \\ ${ }^{4}$ Department of Astronomy, University of Minnesota, 116 Church St SE, Minneapolis, MN 55455, USA; chelsea@ astro.umn.edu \\ ${ }^{5}$ Center for Astronomy and Space Science, University of California-San Diego, 9500 Gilman Drive, Department 0424, San Diego, CA 92093, USA; \\ dharker@ucsd.edu. \\ Received 2009 January 27; accepted 2009 March 18; published 2009 April 21
}

\begin{abstract}
We report Spitzer Space Telescope observations of comet 67P/Churyumov-Gerasimenko at 5.5 and 4.3 AU from the Sun, post-aphelion. Comet 67P is the primary target of the European Space Agency's Rosetta mission. The Rosetta spacecraft will rendezvous with the nucleus at heliocentric distances similar to our observations. Rotationally resolved observations at 8 and $24 \mu \mathrm{m}$ (at a heliocentric distance, $r_{h}$, of $4.8 \mathrm{AU}$ ) that sample the size and colortemperature of the nucleus are combined with aphelion $R$-band light curves observed at the Very Large Telescope (VLT) and yield a mean effective radius of $2.04 \pm 0.11 \mathrm{~km}$, and an $R$-band geometric albedo of $0.054 \pm 0.006$. The amplitudes of the $R$-band and mid-infrared light curves agree, which suggests that the variability is dominated by the shape of the nucleus. We also detect the dust trail of the comet at 4.8 and $5.5 \mathrm{AU}$, constrain the grain sizes to be $\lesssim 6 \mathrm{~mm}$, and estimate the impact hazard to Rosetta. We find no evidence for recently ejected dust in our images. If the activity of $67 \mathrm{P}$ is consistent from orbit to orbit, then we may expect the Rosetta spacecraft will return images of an inactive or weakly active nucleus as it rendezvous with the comet at $r_{h}=4 \mathrm{AU}$ in 2014 .
\end{abstract}

Key words: comets: individual (67P/Churyumov-Gerasimenko) - infrared: solar system - meteors, meteoroids

\section{INTRODUCTION}

Ecliptic comet 67P/Churyumov-Gerasimenko (67P) is the primary target of the Rosetta mission, which will rendezvous with the nucleus in 2014 when the comet has passed aphelion and is $4 \mathrm{AU}$ from the Sun (aphelion distance 5.71 AU). Rosetta will orbit with the comet through perihelion in 2015 (perihelion distance $1.25 \mathrm{AU}$ ). The Rosetta spacecraft is designed to characterize the comet nucleus (morphology, composition) and coma (development of activity, dust-gas interaction, interaction with the solar wind), with both orbiting and landing spacecraft (Glassmeier et al. 2007). Understanding the comet's gas and dust behavior near aphelion is important for mission planning.

In previous perihelion passages, the comet was not characterized over the orbital arc at which the Rosetta spacecraft will rendezvous with the nucleus (Agarwal et al. 2007b). Dynamical models by Fulle et al. (2004) of the dust coma and tail of 67P observed near the perihelion in 2002-2003 have indicated that the comet is significantly active and able to eject millimeter and centimeter-sized grains at 3.6 AU, post-aphelion (i.e., pre-perihelion). However, this conclusion is disputed by Agarwal et al. (2007b), who point out that the dust tail analyzed by Fulle et al. (2004) contains overlapping contributions from all dust ejected before the comet reached 1.5 AU. Direct imaging of the comet with ground-based observations has shown that the comet is active at 2.0 AU, post-aphelion (Agarwal et al. 2007b). Recent ground-based optical and Spitzer Space Telescope mid-infrared (mid-IR) observations have extended that limit to 2.9-3.0 AU (Kadota et al. 2008; Wooden et al. 2008). Some ecliptic comets are active at more than $4 \mathrm{AU}$ from the Sun, e.g., comets $65 \mathrm{P} / \mathrm{Gunn}, 74 \mathrm{P} / \mathrm{Smirnova-Chernykh,} \mathrm{152P/Helin-}$

\footnotetext{
6 Current address: Department of Astronomy, University of Maryland, College Park, MD 20742-2421, USA; msk@ astro.umd.edu
}

Lawrence, and 103P/Hartley (Lowry \& Fitzsimmons 2001; Lowry et al. 1999; Snodgrass et al. 2008). Indeed, even comet $67 \mathrm{P}$ was brighter than expected for a bare nucleus and dust trail in Spitzer spectra of the comet at a heliocentric distance $\left(r_{h}\right)$ of 4.98 AU, pre-aphelion (Kelley et al. 2006), and in ground-based CCD observations at 4.9 AU, pre-aphelion (Mueller 1992; Lamy et al. 2006) — equivalent post-aphelion observations have only recently been presented (Tubiana et al. 2008). The present study of comet 67P is motivated by the lack of aphelion observations and the importance of the characterization of the nucleus and comet activity of 67P for the Rosetta mission.

In this paper, we present and discuss Spitzer Space Telescope (Gehrz et al. 2007; Werner et al. 2004) observations of comet 67P at heliocentric distances between 5.5 and $4.3 \mathrm{AU}$, postaphelion-the latter distance is comparable to the $r_{h}$ at which Rosetta will rendezvous with the comet nucleus. In Section 2, we present imaging observations of the comet obtained with both the Multiband Imaging Photometer for Spitzer (MIPS; Rieke et al. 2004) at $24 \mu \mathrm{m}$ and the Infrared Array Camera (IRAC; Fazio et al. 2004) at $8 \mu \mathrm{m}$, and 14-35 $\mu \mathrm{m}$ spectra of the nucleus with the Infrared Spectrograph (IRS; Houck et al. 2004). In 24 $\mu \mathrm{m}$ MIPS images, we detect the comet's dust trail (Sykes \& Walker 1992) and a tail composed of large grains. We constrain the largest grain size ejected by $67 \mathrm{P}$ to be $\lesssim 6 \mathrm{~mm}$, and then estimate the large grain number density and its impact hazard to Rosetta in Section 3.1. Our observations are consistent with an inactive nucleus between 5.5 and $4.3 \mathrm{AU}$, post-aphelion. We present upper limits to emission from dust at 5.5 and $4.8 \mathrm{AU}$ in Section 3.2. In Section 3.3, we derive the infrared (IR) light curve at $4.8 \mathrm{AU}$ by sampling the emission from the nucleus at 11 epochs with consecutive MIPS and IRAC observations. The Spitzer derived light curves of comet 67P constrain the shape, color-temperature, and effective radius of the nucleus. We examine those properties and their behavior with rotational 


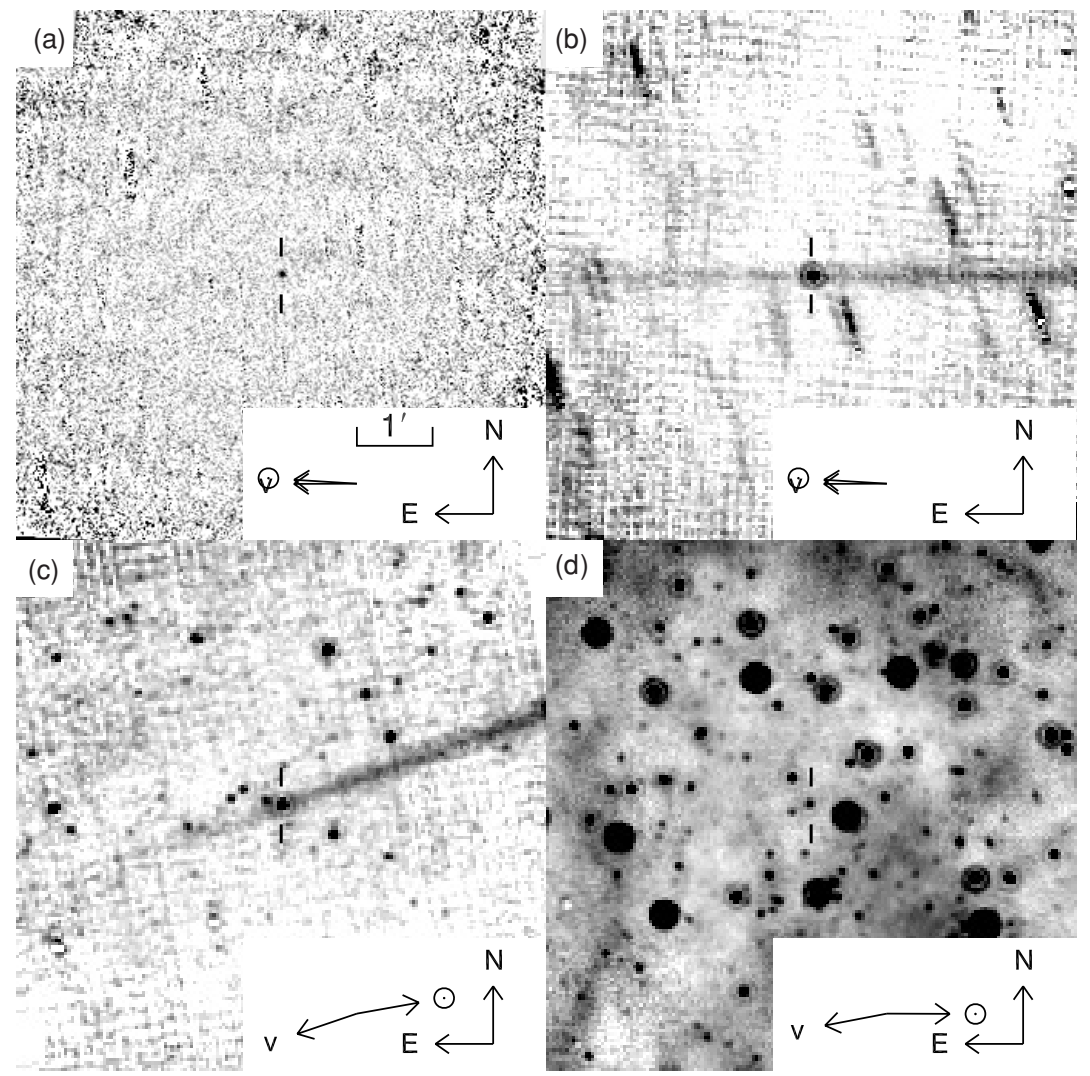

Figure 1. Spitzer images of comet 67P/Churyumov-Gerasimenko. The nucleus is located at the center of all images and marked with vertical bars for clarity. The image scale is provided in (a). North, east, the projected Sun angle $(\odot)$, and the projected comet velocity vector (v) are indicated in the lower right of each image: (a) a co-added mosaic of all 11 IRAC $8 \mu \mathrm{m}$ observations from 2007 May 17 (4.8 AU); (b) a co-added mosaic of all 11 MIPS $24 \mu \mathrm{m}$ images from 2007 May 18 (4.8 AU); (c) the MIPS $24 \mu \mathrm{m}$ image taken on 2006 September 1 (5.5 AU); and (d) a MIPS $24 \mu \mathrm{m}$ image from 2007 September 19 (4.4 AU). The dust trail crosses two of the MIPS images (b and c) along the velocity vector.

phase to show in Section 3.4 that the visible light curve is likely dominated by nucleus shape rather than albedo. However, there are some discrepancies between the visible and IR light curves, therefore some albedo variation cannot be ruled out. Our results are summarized in Section 4.

\section{OBSERVATIONS AND REDUCTION}

\subsection{Imaging}

Spitzer observed comet 67P with the MIPS $24 \mu \mathrm{m}$ instrument in the photometry/super resolution mode (pixel scale 2.55 arcsec pixel $^{-1}$ ). This mode provides 14 dithered images covering an approximate field of view of $7^{\prime} \times 8^{\prime}$ (each image is approximately $5^{\prime} \times 5^{\prime}$ ). The comet nucleus is centered in the dither pattern, and the telescope tracks the comet with its computed nonsidereal rates. Each image has an exposure time of $10 \mathrm{~s}$. The dither pattern was executed nine times for each of the two epochs at $r_{h}=5.5 \mathrm{AU}$ (2006 August 16 and 2006 September 1), three times for each of the 11 epochs at $4.8 \mathrm{AU}$ (2007 May 18), and once for each of the three epochs at 4.4 AU (2007 September 19). The comet is readily identified as a moving point source, centered in the field of view, and bisected by the comet's dust emission (Figure 1), except at 4.4 AU where dust is not observed due to crowding by point sources and diffuse emission arising from the interstellar medium. For the 5.5 and 4.8 AU epochs, we median combined each observation's images and divided each image with the result to remove image artifacts (Spitzer Science Center 2008a) - the 4.4 AU images are too crowed for this step. We stacked the images together in the rest frame of the comet with the MOPEX software (Makovoz \& Khan 2005) to produce two mosaics at 5.5 AU, 11 at $4.8 \mathrm{AU}$, and three at $4.4 \mathrm{AU}$. The 11 MIPS $24 \mu \mathrm{m}$ images at 4.8 AU spanned a duration of 13.17 $\mathrm{hr}$ (start to finish) enabling sampling of the light curve over approximately one rotational period of the nucleus $\left(P_{\text {rot }} \approx 13 \mathrm{hr}\right.$, see Section 3.3). Table 1 presents the observation parameters for the MIPS data.

We also observed comet $67 \mathrm{P}$ with the IRAC $8 \mu \mathrm{m}$ camera in the mapping mode (pixel scale $1.22 \operatorname{arcsec}_{\text {pixel }}{ }^{-1}$ ). The 11 observations spanned a duration of $13.09 \mathrm{hr}$ (start to finish) and, similar to the MIPS observations, measured the light curve just over one rotational period of the nucleus at $r_{h}=4.8 \mathrm{AU}$ (2007 May 17). The observations were designed with a 14-point cyclic dither pattern, and $30 \mathrm{~s}$ exposure times. The images of each observation are mosaicked in the rest frame of the comet with the MOPEX software. The nucleus is faint at $8 \mu \mathrm{m}$ and not readily identifiable. We median combined all 11 images and found the comet to be near the center of the frame (Figure 1). The comet was not detected with the IRAC $4.5 \mu \mathrm{m}$ camera, as expected for a $R=2 \mathrm{~km}$ nucleus at this distance. Table 1 presents the observation parameters for the IRAC data.

Because the IRAC and MIPS instruments are not simultaneously operated, Spitzer observed the two light curves as close in time as was operationally feasible. The IRAC observations were executed first, one rotational period was skipped for instrument calibration and changeover, then the MIPS observations were obtained. We assumed a rotational period of $12.8-12.9 \mathrm{hr}$ to constrain each light curve using approximately equal time steps between each observation. This allows us to phase the two wavelengths ( 8 and $24 \mu \mathrm{m}$ ) together without interpolation. Tubiana et al. (2008) recently constrained the rotational period 
Table 1

Comet 67P/Churyumov-Gerasimenko Observation Log, Photometry, and Effective Radii ${ }^{\mathrm{a}, \mathrm{b}}$

\begin{tabular}{|c|c|c|c|c|c|c|c|c|c|}
\hline AOR Key & $\begin{array}{l}\text { Date } \\
(\mathrm{UT})\end{array}$ & $\begin{array}{c}\text { Time } \\
\text { (hh:mm:ss) }\end{array}$ & $\begin{array}{c}\text { Duration } \\
(\mathrm{min})\end{array}$ & $\phi_{R}$ & $\begin{array}{c}r_{h} \\
(\mathrm{AU}) \\
\end{array}$ & $\begin{array}{c}\Delta_{s} \\
(\mathrm{AU}) \\
\end{array}$ & $\begin{array}{c}\alpha_{s} \\
(\operatorname{deg}) \\
\end{array}$ & $\begin{array}{c}F_{v} \\
(\mathrm{mJy}) \\
\end{array}$ & $\begin{array}{c}R \\
(\mathrm{~km})\end{array}$ \\
\hline \multicolumn{10}{|c|}{ IRAC $7.87 \mu \mathrm{m}$} \\
\hline 21631232 & 2007 May 16 & $23: 55: 28$ & 13.6 & 0.000 & 4.852 & 4.407 & 11.22 & $0.124 \pm 0.021$ & $\cdots$ \\
\hline 21630976 & 2007 May 17 & $01: 21: 41$ & 13.6 & 0.113 & 4.852 & 4.406 & 11.22 & $0.134 \pm 0.014$ & $\ldots$ \\
\hline 21630464 & 2007 May 17 & 03:21:00 & 13.6 & 0.270 & 4.852 & 4.404 & 11.21 & $0.115 \pm 0.014$ & $\cdots$ \\
\hline 21630208 & 2007 May 17 & $05: 14: 31$ & 13.6 & 0.419 & 4.852 & 4.403 & 11.21 & $0.096 \pm 0.013$ & $\cdots$ \\
\hline 21629952 & 2007 May 17 & $05: 56: 08$ & 13.6 & 0.473 & 4.851 & 4.402 & 11.21 & $0.104 \pm 0.014$ & $\ldots$ \\
\hline 21629696 & 2007 May 17 & $07: 14: 17$ & 13.6 & 0.576 & 4.851 & 4.401 & 11.20 & $0.108 \pm 0.013$ & $\cdots$ \\
\hline 21629440 & 2007 May 17 & 08:07:51 & 13.6 & 0.646 & 4.851 & 4.400 & 11.20 & $0.085 \pm 0.010$ & $\cdots$ \\
\hline 21629184 & 2007 May 17 & $09: 25: 59$ & 13.6 & 0.748 & 4.851 & 4.399 & 11.20 & $0.108 \pm 0.014$ & $\ldots$ \\
\hline 21631744 & 2007 May 17 & $10: 19: 31$ & 13.6 & 0.819 & 4.851 & 4.399 & 11.19 & $0.100 \pm 0.014$ & $\cdots$ \\
\hline 21630720 & 2007 May 17 & $11: 44: 04$ & 13.6 & 0.930 & 4.851 & 4.398 & 11.19 & $0.120 \pm 0.014$ & $\cdots$ \\
\hline 21631488 & 2007 May 17 & $12: 52: 03$ & 13.6 & 1.019 & 4.850 & 4.397 & 11.19 & $0.119 \pm 0.014$ & $\ldots$ \\
\hline \multicolumn{10}{|c|}{ MIPS $23.7 \mu \mathrm{m}$} \\
\hline 18555904 & 2006 Aug 16 & $02: 16: 18$ & 30.3 & $\cdots$ & 5.514 & 4.937 & 9.30 & $1.995 \pm 0.049$ & $2.18 \pm 0.18$ \\
\hline 18556416 & 2006 Sep 1 & $01: 56: 47$ & 30.3 & $\ldots$ & 5.488 & 5.149 & 10.44 & $1.750 \pm 0.051$ & $2.08 \pm 0.18$ \\
\hline 21628928 & 2007 May 18 & $01: 34: 42$ & 12.7 & 2.019 & 4.849 & 4.387 & 11.15 & $3.37 \pm 0.11$ & $2.19 \pm 0.17$ \\
\hline 21628672 & 2007 May 18 & 03:03:02 & 12.7 & 2.135 & 4.848 & 4.386 & 11.15 & $3.08 \pm 0.11$ & $2.12 \pm 0.16$ \\
\hline 21628160 & 2007 May 18 & $04: 42: 04$ & 12.7 & 2.265 & 4.848 & 4.385 & 11.14 & $2.97 \pm 0.11$ & $2.06 \pm 0.16$ \\
\hline 21627904 & 2007 May 18 & $06: 48: 40$ & 12.7 & 2.431 & 4.848 & 4.383 & 11.14 & $2.612 \pm 0.079$ & $1.93 \pm 0.15$ \\
\hline 21627648 & 2007 May 18 & $07: 32: 44$ & 12.7 & 2.489 & 4.848 & 4.383 & 11.14 & $2.521 \pm 0.061$ & $1.91 \pm 0.15$ \\
\hline 21627392 & 2007 May 18 & $08: 43: 23$ & 12.7 & 2.582 & 4.848 & 4.382 & 11.13 & $2.502 \pm 0.067$ & $1.90 \pm 0.15$ \\
\hline 21627136 & 2007 May 18 & $09: 47: 50$ & 12.7 & 2.666 & 4.848 & 4.381 & 11.13 & $2.382 \pm 0.081$ & $1.83 \pm 0.14$ \\
\hline 21626880 & 2007 May 18 & $10: 58: 06$ & 12.7 & 2.758 & 4.847 & 4.380 & 11.13 & $2.656 \pm 0.098$ & $1.95 \pm 0.15$ \\
\hline 21626624 & 2007 May 18 & $11: 50: 40$ & 12.7 & 2.827 & 4.847 & 4.379 & 11.12 & $3.018 \pm 0.098$ & $2.05 \pm 0.16$ \\
\hline 21628416 & 2007 May 18 & $13: 25: 44$ & 12.7 & 2.952 & 4.847 & 4.378 & 11.12 & $3.215 \pm 0.098$ & $2.14 \pm 0.17$ \\
\hline 21632000 & 2007 May 18 & $14: 27: 39$ & 12.7 & 3.033 & 4.847 & 4.377 & 11.12 & $3.38 \pm 0.12$ & $2.18 \pm 0.17$ \\
\hline 18557440 & 2007 Sep 19 & $01: 32: 42$ & 7.5 & $\ldots$ & 4.376 & 3.852 & 12.32 & $5.13 \pm 0.41$ & $2.25 \pm 0.19$ \\
\hline 18557696 & 2007 Sep 19 & $01: 41: 56$ & 7.5 & $\cdots$ & 4.376 & 3.852 & 12.32 & $4.62 \pm 0.45$ & $2.22 \pm 0.19$ \\
\hline 18557952 & 2007 Sep 19 & $01: 51: 10$ & 7.5 & $\ldots$ & 4.376 & 3.852 & 12.33 & $4.70 \pm 0.54$ & $2.16 \pm 0.20$ \\
\hline \multicolumn{10}{|c|}{ IRS $14-38 \mu \mathrm{m}$} \\
\hline 18555136 & 2007 Apr 19 & $05: 27: 36$ & 90.2 & $\ldots$ & 4.942 & 4.936 & 11.67 & $\cdots$ & $2.11 \pm 0.20$ \\
\hline 18555392 & 2007 Sep 30 & $18: 19: 58$ & 90.2 & $\ldots$ & 4.325 & 3.974 & 13.34 & $\ldots$ & $1.82 \pm 0.17$ \\
\hline
\end{tabular}

Notes.

${ }^{a}$ Table columns: AOR Key: Spitzer astronomical observation request identifier; time: start of observation; duration: length of observation; $\phi_{R}$ : rotational phase at $4.8 \mathrm{AU}$, using a $12.7047 \mathrm{hr}$ rotation period; $r_{h}$ : heliocentric distance; $\Delta_{s}$ : comet-Spitzer distance; $\alpha_{s}$ : Sun-comet-Spitzer angle; $F_{\nu}$ : flux density; and $R$ : effective radius using the NEATM with an IR-beaming parameter of $0.68 \pm 0.06$.

${ }^{b}$ Instrument absolute calibration errors (3\% for IRAC, $4 \%$ for MIPS, and $10 \%$ for IRS) are not included in the photometric errors to facilitate point-to-point comparisons. Calibration errors are incorporated into the effective radius error.

to be $12.7047 \pm 0.0011 \mathrm{hr}$ at aphelion. Using this new period, the phase offsets between individual IRAC and MIPS observations are minimal and range from 0.005 to 0.022 rotational periods, with a median offset of 0.014 . Our light-curve sampling of 67P differs from the approach of Lamy et al. (2008) who observed the comet at $24 \mu \mathrm{m}$ with Spitzer 16 times-each observation was separated by $0.5,1.0$, or $1.5 \mathrm{hr}$, spanning a total of $\approx 12.5 \mathrm{hr}$.

\subsection{Spectra}

We obtained two spectra of comet $67 \mathrm{P}$ with the IRS long wavelength (14-35 $\mu \mathrm{m})$, low-resolution $(\lambda / \Delta \lambda \approx 100)$ module. One spectrum was taken while 67P was 4.9 AU from the Sun, and the other at $4.3 \mathrm{AU}$ from the Sun. The parameters of the IRS observations are presented in Table 1. The default IRS Stare observing template was used, which placed the comet at two positions separated by $55^{\prime \prime}$ in the $11^{\prime \prime}$ wide by $168^{\prime \prime}$ long slit. Spitzer positioned the slit at the comet's predicted ephemeris coordinates, and followed the comet with its computed nonsidereal rates. The observations were repeated nine times for a total of 18 spectra. We differenced the two slit positions to remove the background, then replaced bad pixels with the average value of the nearest neighbors or ignored them altogether. We extracted spectra from the images using the Spitzer IRS Custom Extraction (SPICE) software package. The profile of the comet is equivalent to a point source, therefore we utilized the default point-source aperture provided by SPICE, which is $7.2 \times \lambda / 27.0 \mu \mathrm{m}$ pixels wide (pixel scale $5^{\prime \prime} .1$ pixel $^{-1}$ ). The individual spectra are averaged together to produce two final spectra, one at each of 4.9 AU and 4.3 AU. The spectra, smoothed with a five-point statistically weighted average, are presented in Figure 2.

\section{RESULTS AND DISCUSSION}

\subsection{The Dust Environment}

\subsubsection{An Empirical Model}

Understanding the near-nucleus dust environment of $67 \mathrm{P}$ when Rosetta will rendezvous with the comet is important for mission planning. Our $24 \mu \mathrm{m}$ images contain emission

\footnotetext{
7 SPICE is available at http://ssc.spitzer.caltech.edu/
} 


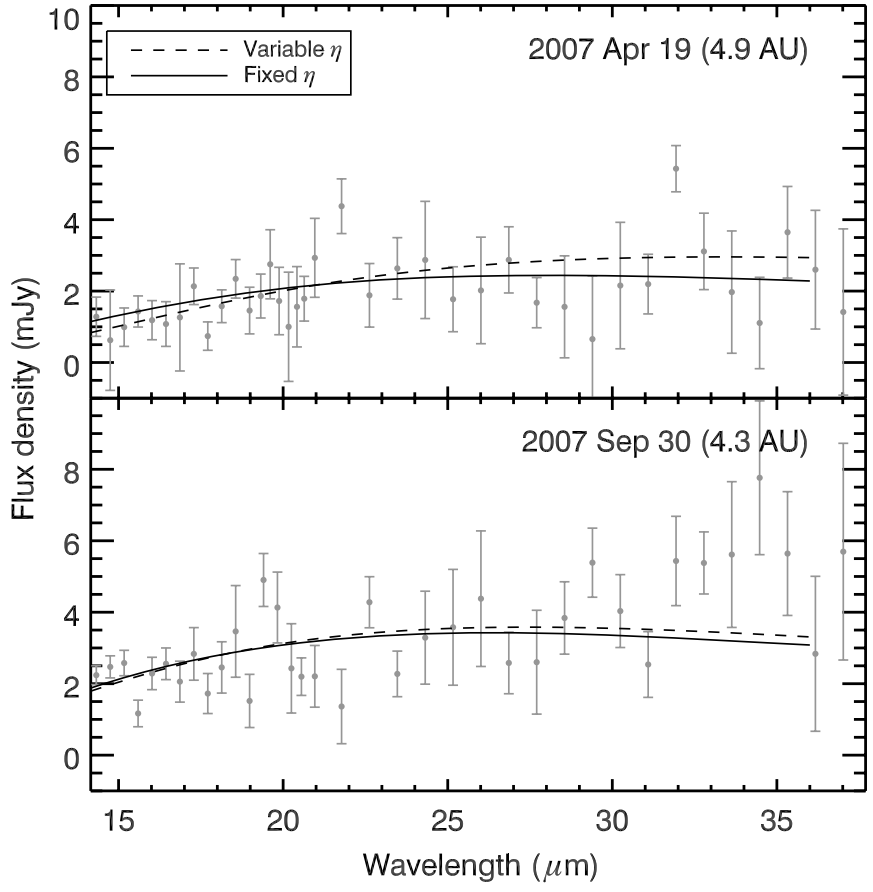

Figure 2. Spitzer IRS 14-38 $\mu \mathrm{m}$ spectra (circles) of comet 67P taken at $r_{h}=4.9$ and $4.3 \mathrm{AU}$. The spectral resolution has been reduced with a fivepoint statistically weighted average. Two best-fit thermal models are shown for each spectrum, one with a variable IR-beaming parameter $(\eta)$, and one with the IR-beaming parameter fixed at $0.68 \pm 0.06$. At $4.9 \mathrm{AU}: R=3.0 \pm 0.5$, $\eta=1.3 \pm 0.4, \chi_{v}^{2}=0.62$ (dashed line), and $R=2.11 \pm 0.17, \eta=0.68 \pm 0.06$, $\chi_{v}^{2}=0.78$ (solid line). At 4.3 AU, $R=1.99 \pm 0.19, \eta=0.80 \pm 0.14, \chi_{v}^{2}=0.62$ (dashed line), and $R=1.82 \pm 0.14, \eta=0.68 \pm 0.06, \chi_{v}^{2}=0.78$ (solid line).

from large dust grains. With photometry of this dust, we can estimate the number density of large grains near the nucleus and assess their impact hazard to spacecraft. Furthermore, if we can adequately describe the morphology of the dust thermal emission it can be removed from the images prior to the measurement of the comet nucleus. To remove the dust, we fit surface brightness profiles to linear cuts $(3 \times 51$ pixels $)$ measured perpendicular to the projected velocity vector of the comet, then subtract our best-fit model from the images. The assumed profile is the sum of a Gaussian function (the dust) and a linear term (the image background). The results of our empirical model fits to the $r_{h}=4.8$ and 5.5 AU images are presented in Figure 3 and Table 2. The photometry has been color-corrected $(\times 1.057)$ to the MIPS $24 \mu \mathrm{m}$ central wavelength of $23.7 \mu \mathrm{m}$ using a blackbody spectral template with $T_{d}=300 r_{h}^{-0.5} \mathrm{~K}$, where $T_{d}$ is the typical dust effective temperature of trail grains (Reach et al. 2009; Sykes \& Walker 1992). Two trends are evident in the fits to the dust surface brightness: (1) the peak surface brightness linearly varies with distance from the nucleus and (2) within the uncertainties $( \pm$ a few degrees in position angle) the dust emission is aligned with the orbit, as would be expected for a dust trail. To generate model images of the dust, we approximate the morphology with the best-fit parameters described in Table 2. The model dust images are subtracted from the 5.5 and 4.8 AU MIPS images.

\subsubsection{A Dynamical Model}

Previous images of 67P at aphelion show a neck-line tail structure. A neck-line structure is a thin, linear feature caused by the projected alignment of dust grains that were emitted at a true

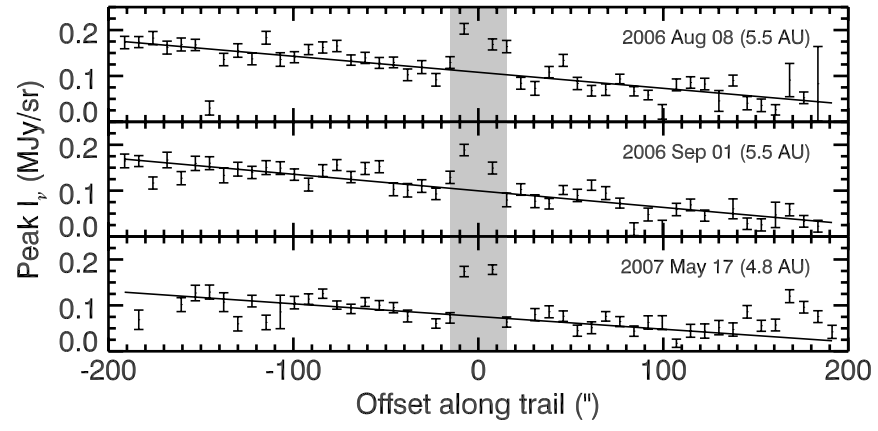

Figure 3. Peak trail surface brightness and best-fit linear trends derived from our empirical trail model fits (Table 2) for three MIPS $24 \mu \mathrm{m}$ epochs. Positive $x$-axis values lie along the projected velocity vector (i.e., these positions are ahead of the comet nucleus). The data points in the grayed area were excluded from the profile fits to prevent the nucleus from affecting the results.

anomaly $180^{\circ}$ prior to the observation in question (Southworth 1963; Kimura \& Liu 1975). The enhancement is strongest when the observer is located near the orbital plane of the comet, which is frequently the case for ecliptic comets such as 67P. Small dust grains cannot remain near the nucleus for many years; therefore, if a neck-line is observed in our Spitzer observations, it must be comprised of large dust grains that weakly interact with solar radiation pressure. Observations of neck-lines are beneficial because they allow us to measure the size distribution of dust grains emitted by the comet. This becomes apparent when we consider that larger dust grains are ejected from the nucleus with lower ejection velocities and are accelerated less effectively by solar radiation pressure than smaller grains. Therefore, the largest grains remain the closest to the nucleus and the grain size decreases with increasing distance along the neck-line. In principle, the variation of the surface brightness distribution of a neck-line with distance from the nucleus can be transformed into a grain size distribution. In the Spitzer MIPS images, the mere existence of a neck-line can help us constrain the largest grains emitted by 67P. The age of a neck-line observed on 2006 August 16 would be 3.9 years, corresponding to dust emitted at a post-perihelion distance of $r_{h}=1.26 \mathrm{AU}$. Similarly, if a neck-line were observed on 2007 May 18, its age would be 4.6 years, i.e., released from the nucleus at $r_{h}=1.30 \mathrm{AU}$, post-perihelion.

In order to determine if a neck-line tail structure exists in our images, we compute simulated images of the comet using the dynamical dust model of Kelley et al. (2008). The primary forces acting on a dust grain are the force from solar radiation $\left(F_{r}\right)$, the force of solar gravity $\left(F_{g}\right)$, and gravitational perturbations from the planets. Dynamical models of comets typically describe dust grains with the parameter $\beta=F_{r} / F_{g}$. Both forces vary with $r_{h}^{-2}$, therefore $\beta$ is independent of heliocentric distance. The $\beta$ parameter does, however, depend on grain size:

$$
\beta=\frac{F_{r}}{F_{g}}=\frac{0.57 Q_{\mathrm{pr}}}{\rho a}
$$

where $Q_{\mathrm{pr}}$ is the radiation pressure efficiency $(\approx 1$ for large grains), $\rho$ is the grain density in units of $\mathrm{g} \mathrm{cm}^{-3}$, and $a$ is the grain radius in units of $\mu \mathrm{m}$ (Burns et al. 1979). When converting from $\beta$-values to grain radii, we follow Kelley et al. (2008) and adopt a grain density of $1 \mathrm{~g} \mathrm{~cm}^{-3}$ (see Kelley et al. 2008 for a discussion related to this choice). To describe the dust ejection dynamics and history of comet 67P, we start with the best parameters of Kelley et al. (2008). In summary, Kelley et al. (2008) fit 
Table 2

Empirical Model Parameters Used to Describe the Dust Trail Morphology and Brightness ${ }^{\mathrm{a}}$

\begin{tabular}{|c|c|c|c|c|c|c|}
\hline \multirow{2}{*}{$\begin{array}{l}\text { Observation } \\
\text { Date }\end{array}$} & \multicolumn{2}{|c|}{$A\left(\mathrm{MJy} \mathrm{sr}^{-1}\right)^{\mathrm{b}}$} & \multicolumn{2}{|c|}{$\mu\left(10^{3} \mathrm{~km}\right)$} & \multicolumn{2}{|c|}{$\sigma\left(10^{3} \mathrm{~km}\right)$} \\
\hline & $m$ & $b$ & $m$ & $b$ & $m$ & $b$ \\
\hline 2006 Aug 8 (5.5 AU) & $(-3.5 \pm 0.2) \times 10^{-4}$ & $0.108 \pm 0.002$ & 0.0 & $-1.5 \pm 2.1$ & 0.0 & $24 \pm 1$ \\
\hline 2006 Sep 1 (5.5 AU) & $(-3.6 \pm 0.2) \times 10^{-4}$ & $0.100 \pm 0.002$ & 0.0 & $-1.5 \pm 1.1$ & 0.0 & $24 \pm 2$ \\
\hline
\end{tabular}

Notes.

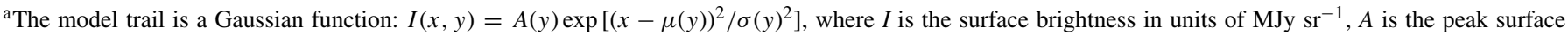
brightness in units of $\mathrm{MJy} \mathrm{sr}^{-1}, \mu$ is the trail offset from the projected orbit in units of $\mathrm{km}, \sigma$ is the trail width in units of km, and $x$ and $y$ are, respectively, offsets perpendicular and parallel to the projected orbit, measured in units of arcseconds. Each parameter, $A, \mu$, and $\sigma$, is a linear function, e.g., $A=m y+b$.

${ }^{\mathrm{b}}$ The uncertainties in parameter $A$ do not include the MIPS absolute calibration error of $4 \%$.

Spitzer MIPS images of the comet taken on 2004 February 23 (4.5 AU, pre-aphelion) and found three distinct dynamical components: (1) a dust trail with an age greater than one orbit, (2) a neck-line ejected near the 2002 perihelion passage, and (3) a broad dust tail. The surface brightness distribution of the dust in the $4.5 \mathrm{AU}$, pre-aphelion, image was best modeled by:

1. a dust production proportional to $\cos z_{\odot}$, where $z_{\odot}$ is the Sun-zenith angle of a surface element ejecting dust grains, 2. a size distribution proportional to $a^{-3.5}$,

3. an ejection velocity equal to $0.5 \beta^{0.5} r_{h}^{-0.5} \cos z_{\odot} \mathrm{km} \mathrm{s}^{-1}$, and

4. dust grains with $-5 \leqslant \log \beta \leqslant-1(6 \mu \mathrm{m} \lesssim a \lesssim 60 \mathrm{~mm})$.

Kelley et al. (2008) assumed that the comet's dust production is proportional to dust production rates inferred from optical photometry (Kidger 2003), $Q_{d} \propto r_{h}^{-5.8}$. We note that the above parameters are similar to those of Ishiguro (2008), derived by comparison of dynamical models to three epochs of groundbased optical observations. The greatest differences between the Kelley et al. (2008) and Ishiguro (2008) models are the choice of the largest grain sizes $(60 \mathrm{~mm}$ versus $5 \mathrm{~mm})$ and dust production rates $\left(Q_{d} \propto r_{h}^{-5.8}\right.$ vs. $\left.r_{h}^{-3}\right)$.

We generated two simulations of 67P using the dynamical model and best parameters of Kelley et al. (2008), with the added stipulation that no grains are ejected outside of $r_{h}=4.5 \mathrm{AU}$. This distance corresponds to the comet-Sun distance during the 2004 February Spitzer observations, where little to no coma was observed (Kelley et al. 2008; Lamy et al. 2008). The simulations reproduce the observing conditions of our 5.5 AU and 4.8 AU images. Each simulation tracked $\approx 2 \times 10^{6}$ grains and recorded their final positions, which are projected onto the celestial sphere for an observer located at Spitzer. The oldest grains tracked were ejected near aphelion in 1986 March, and during the simulation, the comet passed perihelion three times. The resulting images are convolved with a model $24 \mu \mathrm{m}$ point-spread function (PSF) generated with version 2.0 of the Tiny Tim/Spitzer software. ${ }^{8}$ In Figures 4 and 5, we present the simulated $24 \mu \mathrm{m}$ images and surface brightness profiles, generated with a variety of $\beta$-value cutoffs: (a) $\beta>1 \times 10^{-5}$, (b) $\beta>5 \times 10^{-5}$, (c) $\beta>1 \times 10^{-4}$, (d) $\beta>2 \times 10^{-4}$, (e) $\beta>5 \times 10^{-4}$. Simulations (c) and (d) best reproduce the observed profile at both epochs. We estimate that $67 \mathrm{P}$ ejects grains with $\beta$-values larger than $1 \times 10^{-4}$, corresponding to grains smaller than $\approx 6 \mathrm{~mm}$ in radius. This is essentially the same size upper limit derived by Ishiguro (2008).

\footnotetext{
8 Tiny Tim/Spitzer is available from the Spitzer Science Center http://ssc.spitzer.caltech.edu/
}

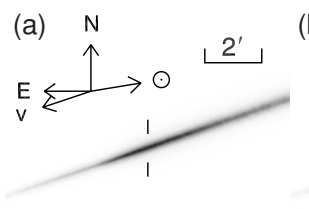

(b)

(c)

(d)

(e)
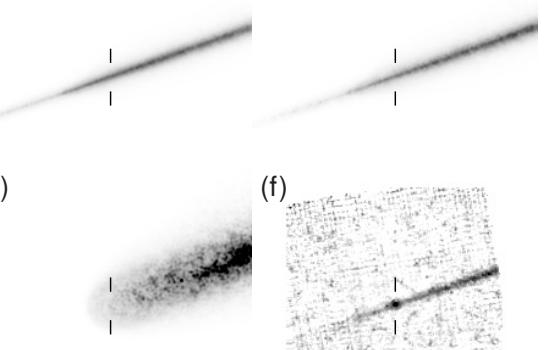

(f)
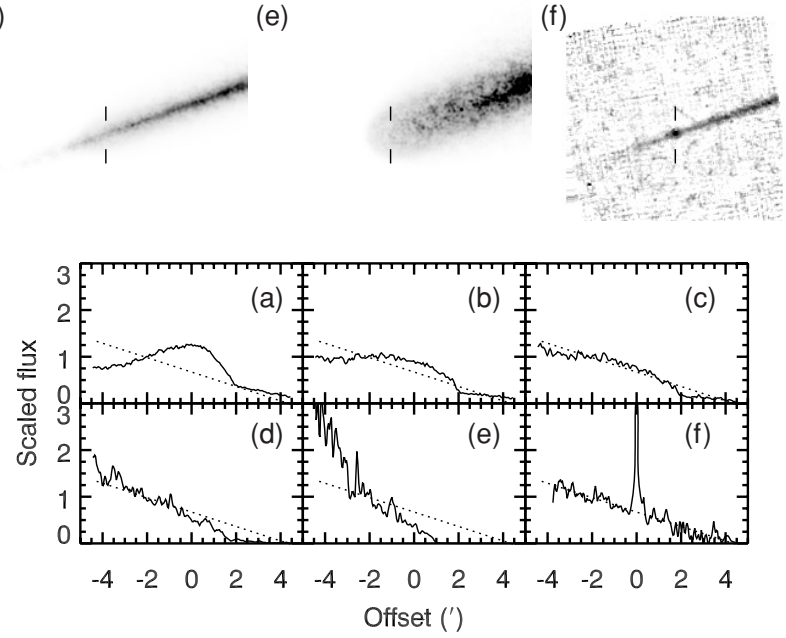

Figure 4. Top: simulated $24 \mu \mathrm{m}$ images (a-e) and the 2006 August 16 MIPS image (f). Each simulated image was generated with a different large grain cutoff: (a) $\beta>1 \times 10^{-5}$, (b) $\beta>5 \times 10^{-5}$, (c) $\beta>1 \times 10^{-4}$, (d) $\beta>2 \times 10^{-4}$, (e) $\beta>5 \times 10^{-4}$. All images are scaled to a value of 1.0 at a distance of $2^{\prime}$ behind the nucleus. North is up, east is to the left, the projected Sun angle $(\odot)$ and comet velocity vector (v) are indicated with arrows, and the image scale is provided in (a). The vertical lines mark the location of the nucleus. Bottom: surface brightness profiles generated from each of the simulated images (a-e) and the observation (f). We fit a linear profile to the observed dust in (f), and plot it as a dotted line in each subplot.

Formally, a neck-line tail structure is present in our Spitzer observations. The neck-line and dust trail overlap in both the 5.5 $\mathrm{AU}$ and 4.8 $\mathrm{AU}$ images, and they cannot be resolved into two components as has been observed by Kelley et al. (2008), Ishiguro (2008), and Agarwal et al. (2007a). The distinction between neck-line and trail in our observations rests merely on their dynamical parameters, since their grain populations have approximately the same sizes $(\sim 1 \mathrm{~mm}$ in radius) at these heliocentric distances. Taking into account our $\beta$-value lower limit of $10^{-4}$, the neck-line is located at a position angle $\approx 1^{\circ}$ north (at 5.5 AU) or south (at $4.8 \mathrm{AU}$ ) of the trail. In our images, we find that $15 \%-25 \%$ of the observed flux within 6.5 of 67P's projected orbit is attributable to the neck-line tail, released at $r_{h} \approx 1.3 \mathrm{AU}$. 


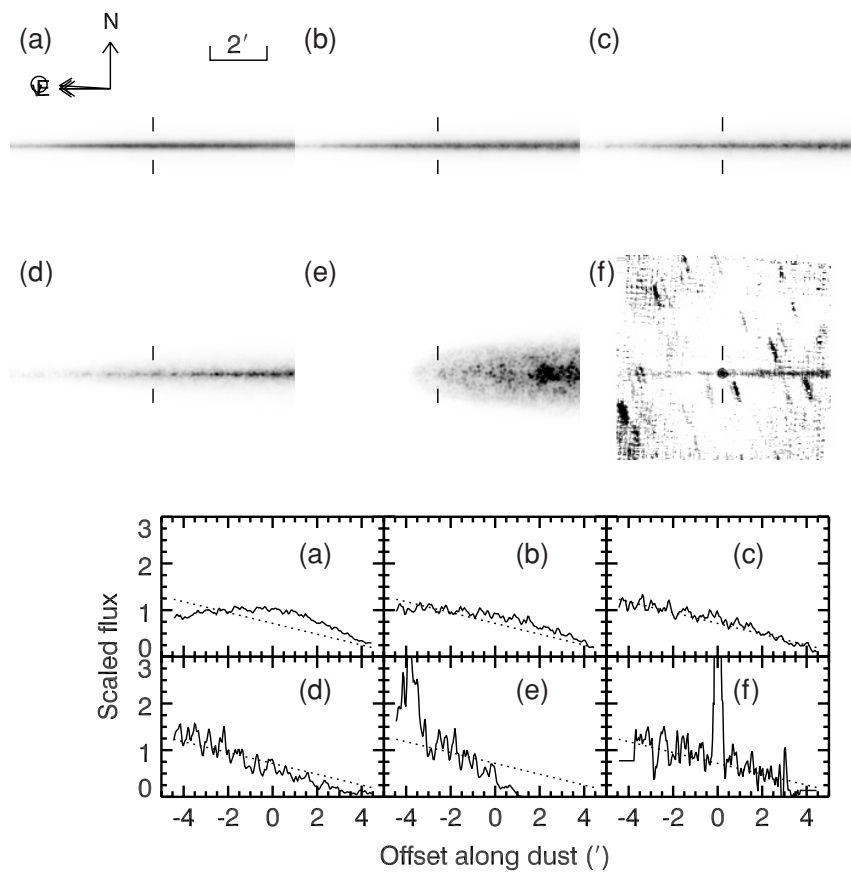

Figure 5. Same as Figure 4, except the 2007 May 18 epoch simulations and MIPS image are shown.

\subsubsection{Large Dust Grain Number Density and Impact Hazard to Rosetta}

Kelley et al. (2008) estimate the trail surface brightness near the nucleus to be $\sim 0.1 \mathrm{MJy} \mathrm{sr}^{-1}$ in $24 \mu \mathrm{m}$ MIPS images obtained at 4.5 AU pre-aphelion. Their estimate depends on a dynamical model to separate trail emission from tail emission. Kelley et al. (2008) then derive a number density of $\sim 10^{-11} \mathrm{~m}^{-3}$ for millimeter-sized grains. From our empirical model (Table 2), the peak dust surface brightness at the nucleus is $0.076 \pm 0.004$ MJy $\mathrm{sr}^{-1}$ and the full width at half-maximum (FWHM) is $(19 \pm 2) \times 10^{3} \mathrm{~km}$ at $4.8 \mathrm{AU}$, post-aphelion. These values are improvements over the Kelley et al. (2008) analysis because the dust width and surface brightness may be measured closer to the nucleus. Moreover, we can estimate the extent of the dust along the Spitzer line of sight using the above dynamical simulations. Figure 6 shows the 4.8 AU simulation as viewed by an observer located above the ecliptic plane. The apparent width of the dust along Spitzer's line of sight is $\approx 60,000 \mathrm{~km}$.

Assuming the dust is entirely comprised of millimeter-sized grains with a dust temperature of $T_{d}=300 r_{h}^{-0.5} \mathrm{~K}$ and contained within a volume of $38,000 \times 60,000 \times 40,000 \mathrm{~km}$, we derive a grain number density of $(1.33 \pm 0.06) \times 10^{-12} \mathrm{~m}^{-3}$. Our assumed volume corresponds to the observed width of the trail $(2 \sigma$, Table 2$)$, the thickness of the simulated dust along the line of sight in the $\beta>10^{-4}$ model, and the MIPS spatial resolution. The number density is about a factor of 10 smaller than the estimate of Kelley et al. (2008). We caution that the uncertainty is only based on the observed flux, and we are assuming that our chosen simulation parameters accurately represent the behavior of the comet.

Inspection of Figure 6 reveals that a spacecraft, such as Rosetta, approaching from the interior of the comet's orbit will not encounter the bulk of the dust. To estimate an upper limit to Rosetta's impact hazard based on our simulations and observations, we assume that the dust grains are uniformly distributed in space, and the Rosetta's path length through that

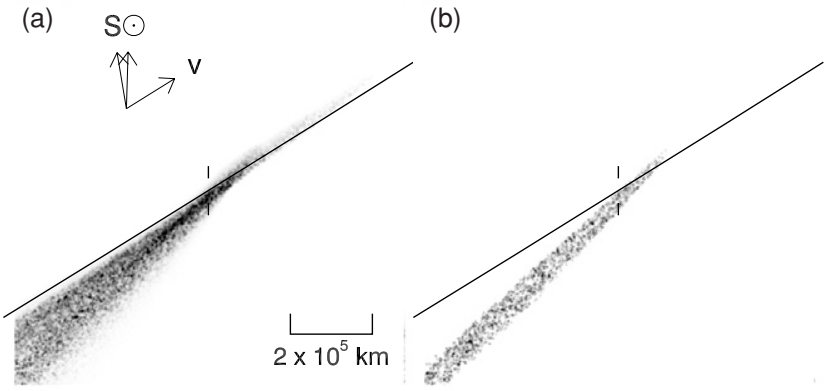

Figure 6. Simulated $24 \mu \mathrm{m}$ images of 67P's 2007 May 18 dust environment as viewed by an observer located above the ecliptic plane: (a) all dust with $\beta>10^{-4}$; and (b) neck-line tail dust with $\beta>10^{-4}$. A solid line marks the projected orbit of the comet, the vertical lines mark the location of the nucleus the projected Sun angle $(\odot)$, comet velocity vector $(\mathrm{v})$, and Spitzer direction $(\mathrm{S})$ are indicated with the arrows, and the image scale is provided in (a). The surface brightness of image (b) has been scaled by a factor of 2 relative to image (a) to improve contrast, and both images have been convolved with a $4600 \mathrm{~km}$ wide (FWHM) Gaussian kernel. The neck-line tail grains and other dust ejected near perihelion have orbits that are distinctly different from the trail grains, which are located closer to the orbit of the comet.

dust is $30,000 \mathrm{~km}$ (half of the dust's thickness in the comet's orbital plane). The total impact probability to millimeter-sized grains is $<0.004 \% \mathrm{~m}^{-2}$. Taking the spacecraft cross-section to be $4 \mathrm{~m}^{2}$ and the total solar panel cross-section to be $64 \mathrm{~m}^{2}$ (Glassmeier et al. 2007), Rosetta has $<0.3 \%$ chance of encountering a millimeter-sized grain during approach to the nucleus. During comet flyby missions, spacecraft typically survive collisions with a few $0.1-1 \mathrm{mg}(\approx 500 \mu \mathrm{m})$ dust grains at high relative speeds, $\sim 1 \mathrm{~km} \mathrm{~s}^{-1}$ (A'Hearn et al. 2005; Green et al. 2004; McDonnell et al. 1987, 1993). However, previous flyby missions visited their respective comets during vigorous coma activity, which enhances the density of large grains. Therefore, our encounter probability upper limit is not unusual and seems reasonable.

\subsection{Comet Activity}

The Spitzer MIPS observations are suitable for a search for faint coma emission. We restricted our search to the MIPS images because they are more sensitive to emission from dust at 4-6 AU from the Sun. First, the 11 MIPS observations at 4.8 AU are averaged together to create a deep image, then the empirical dust model is subtracted from the images. Subtracting the dust model should not affect our search for a dust coma as comae are peaked near the nucleus yet the observed dust varies linearly across the position of the nucleus (Figure 3). The nucleus of 67P has an FWHM of 2.2 pixels, equivalent to the FWHM of point sources in the surrounding field of view when the images are mosaicked in the celestial reference frame. A comparison of a model PSF generated with Tiny Tim/Spitzer $(T=200 \mathrm{~K})$ to the observed radial profile of $67 \mathrm{P}$ (Figure 7) strongly suggests that the nucleus is devoid of an extensive coma at this heliocentric distance. The azimuthally averaged coma flux at $6-8$ pixels (15$\left.20^{\prime \prime}\right)$ from the nucleus i.e., outside of the first diffraction ring of a point source, is $0.0025 \pm 0.0030 \mathrm{MJy} \mathrm{sr}^{-1}$, yielding a $1 \sigma$ upper limit of $0.0055 \mathrm{MJy} \mathrm{sr}^{-1}$. Similarly, we also measured a stellar profile and a coma upper limit of $0.0042 \mathrm{MJy} \mathrm{sr}^{-1}$ in the images at 5.5 AU.

We can convert the surface brightness upper limits, $I(\rho)$, to an integrated coma brightness upper limit if we assume a coma shape. We assume a steady-state coma surface brightness distribution of $\rho^{-1}$, where $\rho$ is the angular distance from the nucleus. This assumption is typical for photometry of distant 


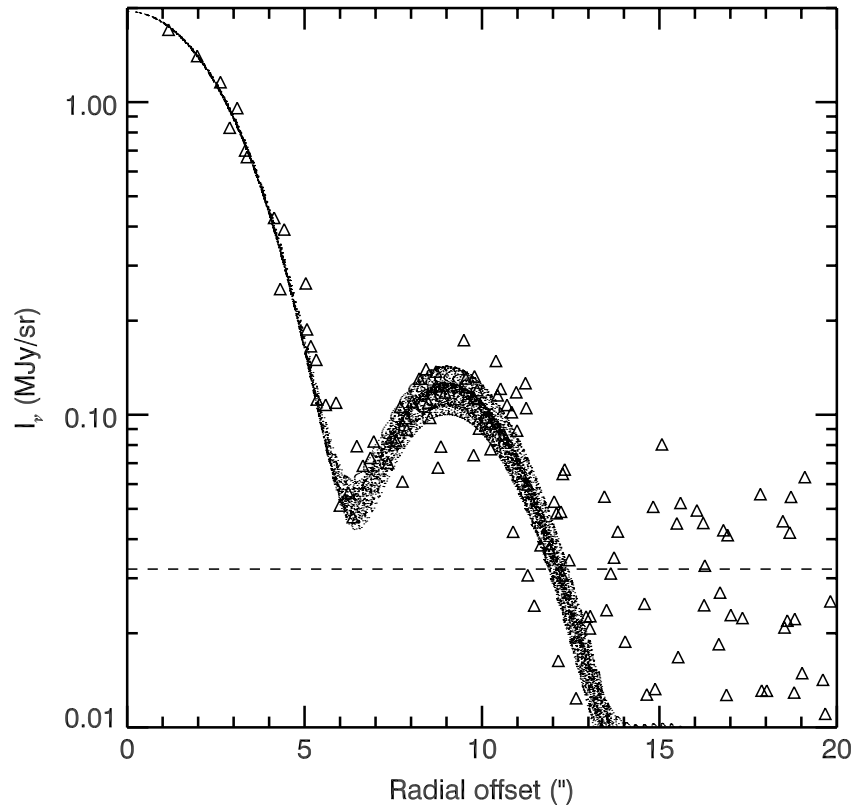

Figure 7. $24 \mu \mathrm{m}$ radial surface brightness profile (triangles) of comet 67P/Churyumov-Gerasimenko at 4.8 AU (2007 May 18) after emission from the dust trail has been removed. A $200 \mathrm{~K}$ model PSF is plotted with dots, and the $1 \sigma$ background level is marked with a horizontal dashed line. There is no evidence for a dust coma in this or any other image.

comets (e.g., Snodgrass et al. 2008). The more steeply shaped profile of a radiation pressure dominated coma, $I_{v} \propto \rho^{-1.5}$ (Jewitt \& Meech 1987), has a less restrictive upper limit by a factor of 2. The integrated coma brightness upper limits at $24 \mu \mathrm{m}$ are computed from $F_{C} \leqslant 2 \pi \rho^{2} I(\rho)$, and are equal to $0.26 \mathrm{mJy}$ at $r_{h}=4.8 \mathrm{AU}$, and $0.20 \mathrm{mJy}$ at $5.5 \mathrm{AU}$. These upper limits are $9 \%$ of the point-source fluxes presented in Section 3.3. Removing a $9 \%$ coma contribution from our photometry decreases our best-fit nucleus radii by $19 \%$. In Section 3.3, we assume that the coma contribution is zero, and find that the resulting nucleus radii agree with previous estimates.

\subsection{The Mid-Infrared Light Curve and the Nucleus Size, Shape, and Temperature}

\subsubsection{Photometry}

The MIPS and IRAC light curves allow us to constrain the surface temperature distribution of the nucleus which is required in order to accurately model its size and shape. First, we describe our techniques for measuring the comet flux in our light-curve observations at $4.8 \mathrm{AU}$; next, we describe our techniques for the snapshot observations at 4.4 and $5.5 \mathrm{AU}$; and finally, we describe our nucleus thermal model and the results of the model fits to the data.

For the images taken at 4.8 AU, we subtract the empirically derived model dust from each mosaicked MIPS observation. Next, we subtract the celestial background. As the telescope tracks the comet, background objects approach and recede from the vicinity of the nucleus. To remove the celestial objects, we identify image pairs where the comet has moved at least 6 pixels. Aligning and differencing these image pairs removes the fixed background objects. Finally with aperture photometry, we derive the flux of the comet utilizing for IRAC a 5 pixel source aperture, and a 10-25 pixel background annulus. The aperture correction for the IRAC photometry is $\times 1.082$, and the color-correction is $\times 0.869$. For MIPS the aperture and color-

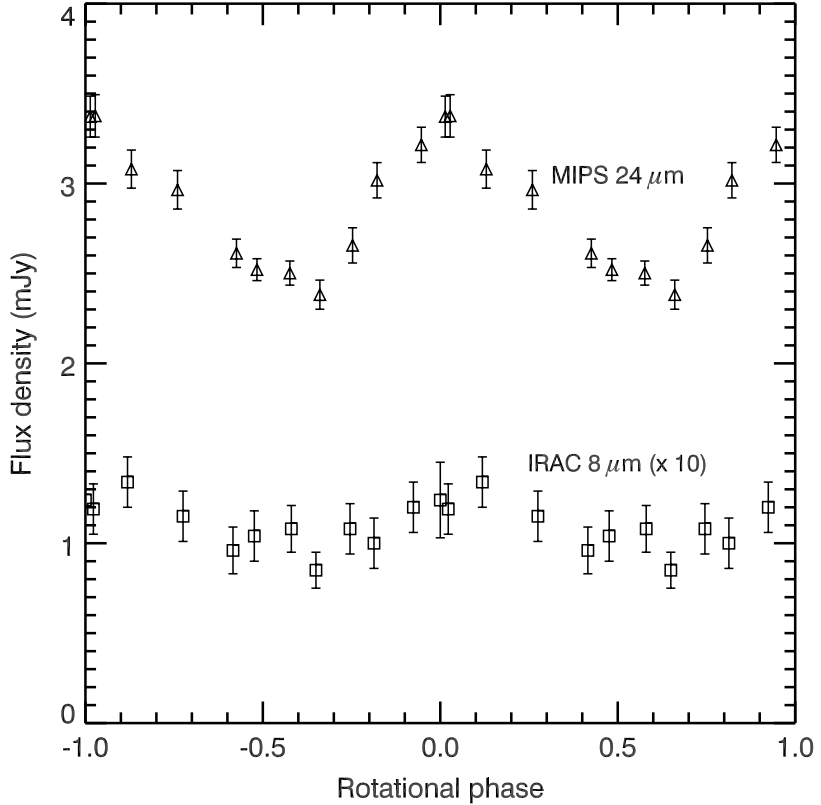

Figure 8. Spitzer MIPS $24 \mu \mathrm{m}$ (triangles) and IRAC $8 \mu \mathrm{m}$ (squares) light curves, phased with a rotational period of $12.7047 \mathrm{hr}$. The IRAC light curve has been scaled by a factor of 10 .

corrections are, respectively, $\times 1.180-1.349$ and $\times 1.047$ for $4-6$ pixel source apertures and a 15-30 pixel background aperture. The color-corrections were generated and iteratively refined using a best-fit near-Earth asteroid thermal model (NEATM; Harris 1998) spectrum (see below), as prescribed by the Spitzer Science Center (2006, 2008a).

At 4.4 and 5.5 AU, a slightly different approach is required. First, our empirical dust model is subtracted from the $5.5 \mathrm{AU}$ images. At 4.4 AU, the images were not sufficiently sensitive to detect a dust trail, therefore no trail was subtracted. The nucleus of 67P falls very close to a few background stars in all images. The comet was not tracked sufficiently long enough to allow celestial sources to move away from the nucleus therefore the shift and align technique we used to remove point-source contamination in the 4.8 AU images is not possible. Instead, we subtract these stars with a PSF fitting program (Diolaiti et al. 2000). The program does not preserve the critically sampled Spitzer PSF adequately enough for precise photometry (it is designed for oversampled images), but does sufficiently remove background stellar sources fainter than the nucleus for our purposes. We repeat the same photometry procedure as executed on the 4.8 AU data (color-corrections are $\times 1.046$ at $5.5 \mathrm{AU}$ and $\times 1.050$ at $4.4 \mathrm{AU})$

Table 1 and Figure 8 present the color- and aperture-corrected photometry. The light-curve photometry has also been corrected to remove the effects of the changing observation geometry as the distance between the Earth and 67P increased during the 1.5 days that elapsed between the first IRAC and the last MIPS observation ( $\leqslant 1.5 \%$ correction). The absolute calibration errors for MIPS and IRAC are 3\% and 4\%, respectively, but are not included in the reduced photometry in order to enable direct comparison of the point-to-point variation of the data. Absolute calibration errors are included in the thermal model fit results discussed below. The mean $24 \mu \mathrm{m}$ and mean $8 \mu \mathrm{m}$ fluxes at $4.8 \mathrm{AU}$ are $F_{24}=2.83 \pm 0.15 \mathrm{mJy}$ and $F_{8}=0.109 \pm 0.015 \mathrm{mJy}$.

\subsubsection{Nucleus Thermal Model}

To derive 67P's nucleus size and temperature, we adopt the NEATM (Harris 1998), noting that this model assumes the 
nucleus is spherical and in instantaneous equilibrium with insolation. Modeling the nuclei of comets with the NEATM has been successful in the past (Fernández et al. 2000, 2006; Kelley et al. 2006), including a successful reproduction of the global properties of the nucleus of comet 9P/Tempel substantiated by resolved temperature maps created from Deep Impact observations (Groussin et al. 2007). Despite the simplifications of the model, the NEATM can still provide good estimates for the effective radius and albedo of the comet (e.g., see Groussin et al. 2007).

In the NEATM, the temperature distribution of the surface peaks at the subsolar point and decreases with the cosine of the Sun-zenith angle. The hemisphere beyond the solar terminator has a temperature of $0 \mathrm{~K}$. The subsolar temperature is computed via

$$
T_{s s}=\left[\frac{(1-A) S}{\eta \epsilon \sigma}\right]^{1 / 4}(\mathrm{~K}),
$$

where $A$ is the Bond albedo, $S=1365 r_{h}^{-2} \mathrm{~W} \mathrm{~m}^{-2}$ is the solar flux at the distance of the comet ( $r_{h}$ measured in AU), $\eta$ is the IR-beaming parameter, $\epsilon=0.9$ is the infrared emissivity, and $\sigma$ is the Stefan-Boltzmann constant. Commonly, the geometric albedo, $p$, is reported in the literature, rather than the Bond albedo ( $p=A / q$, where $q$ is the phase integral, see Hanner et al. 1981; Bowell et al. 1989). The range of geometric albedos derived for comet nuclei is 2\%-6\% (Lamy et al. 2004), which has a minor impact on surface temperature. $\eta$ remains as the primary unknown quantity. Formally, $\eta$ parameterizes how the thermal emission is preferentially emitted toward the Sun due to surface roughness, but because the NEATM assumes instantaneous equilibrium with insolation, $\eta$ must also account for the thermal inertia of real world surfaces. In practice, $\eta$ is constrained by the shape of the nucleus thermal emission. We derive $\eta$ by iteratively comparing the observed spectral energy distribution to the spectrum of a NEATM modeled hemisphere, observed at the same phase angle. The primary source of uncertainty in $\eta$ is our IRAC $8 \mu \mathrm{m}$ photometry.

Since the thermal emission from comet nuclei is weakly dependent on the surface albedo, we turn to optical measurements to constrain this value. We follow the methods of Russell (1916) and Jewitt (1991), to employ the relationship between radius, absolute visual magnitude, and albedo:

$$
p=\left[\frac{1.496 \times 10^{8}}{R} 10^{0.2\left(M_{\odot}-H\right)}\right]^{2}(\mathrm{~km}),
$$

where $R$ is the effective radius in $\mathrm{km}, p$ is the geometric albedo, $H$ is the absolute optical magnitude of the nucleus and $M_{\odot}$ is the absolute magnitude of the Sun. Using a reduced- $\chi^{2}$ fitting routine, we simultaneously vary $p, \eta$, and $R$ in Equation (3) and the NEATM to constrain these parameters for comet $67 \mathrm{P}$.

The nonsimultaneous IRAC and MIPS light curves must be phased together using a measurement of the nucleus rotation period. The cadence and time span of our Spitzer observations do not permit unique determination of the rotation period of the nucleus. However, other investigators have more appropriate observations. Lamy et al. (2006) observed 67P with the Hubble Space Telescope in $2003 \mathrm{March}$, and constrained the period to $P=12.41 \pm 0.41 \mathrm{hr}$. Lowry et al. (2006) observed the comet over three nights with the NTT in 2005 May, and measured $P=12.72 \pm 0.05 \mathrm{hr}$. Tubiana et al. (2008) have phased together Very Large Telescope (VLT) light curves measured in 2006 May and 2006 August (near aphelion), and determined the period to be $12.7047 \pm 0.0011 \mathrm{hr}$, commensurate with the previously determined periods, although with greater precision. Because of the high precision, and that it was obtained closest in time to our observations, we adopt the aphelion period of $12.7047 \mathrm{hr}$ for our analysis of the Spitzer data.

The variation of the nucleus' color-temperature with rotational phase can be assessed by combining the IRAC and MIPS light curves at 4.8 AU, and fitting a Planck function to each pair of data points. The derived color-temperatures are within $1 \sigma$ of the mean value, $187 \pm 4 \mathrm{~K}$, at all longitudes. Thus, we do not detect any variation in temperature with rotation period.

As discussed in Section 3.4, the aphelion light curves from Tubiana et al. (2008) agree in amplitude with our Spitzer observations. Using our mean fluxes and their mean $R$-band absolute magnitude $\left(H_{R}=15.35 \pm 0.04, M_{\odot, R}=-27.14\right)$, we derive $R=2.04 \pm 0.11 \mathrm{~km}, \eta=0.68 \pm 0.06$, and $p_{R}=0.054 \pm 0.006$, via $\chi^{2}$ minimization. We note that our fit has no degrees of freedom because we are fitting three data points with a three-parameter model. The mean effective radius agrees with other recent measurements, which range from 1.9 to $2.1 \mathrm{~km}$ once differences in albedo, absolute solar magnitude, and adopted phase angle behaviors are taken into account (Lamy et al. 2007; Tubiana et al. 2008; Tubiana 2008). The axial ratio, derived from the MIPS light-curve minimum (2.382 mJy) and maximum ( $3.37 \mathrm{mJy})$, is $a / b \geqslant 1.41 \pm 0.07$, which also agrees with the analyses of Lamy et al. (2006) and Tubiana et al. (2008). The low $\eta$-value strongly suggests that the surface has a low thermal inertia, similar to the surfaces of other comets (Groussin et al. 2007). The best-fit radii from all our MIPS observations, derived assuming $\eta=0.68 \pm 0.06$, are presented in Table 1.

\subsection{Comparison to Other Light Curves}

Lamy et al. (2008) created a $24 \mu \mathrm{m}$ light curve derived from observations of $67 \mathrm{P}$ obtained when the comet was at $4.5 \mathrm{AU}$ from the Sun, pre-aphelion. This latter light curve is compared to our data in Figure 9. We scaled the Lamy et al. (2008) data by

$$
\frac{\operatorname{NEATM}\left(r_{h, 1}, \Delta_{s, 1}, \alpha_{s, 1}, \eta, p_{R}, R\right)}{\operatorname{NEATM}\left(r_{h, 2}, \Delta_{s, 2}, \alpha_{s, 2}, \eta, p_{R}, R\right)}=0.746,
$$

where $\Delta_{s}$ is the comet-Spitzer distance, $\alpha_{s}$ is the Sun-cometSpitzer angle, subscript 1 designates the observing conditions for our MIPS light curve (Table 1), subscript 2 designates the observing conditions for the Lamy et al. (2008) light curve $\left(r_{h}=4.48 \mathrm{AU}, \Delta_{s}=4.04 \mathrm{AU}, \alpha_{s}=12.1\right), \eta=0.68$, and $p_{R}=0.054$. The scale factor is independent of $R$. The absolute phasing of the two light curves is unknown-over 2000 rotational periods have elapsed between the two observations. We have shifted the Lamy et al. (2008) light curve to minimize the point-to-point variation between the two data sets. Only near 0.1 phases do the two light curves significantly disagree, but since both light curves are sparsely sampled at these phases the discrepancies cannot be resolved. Lamy et al. (2008) combine their mid-IR light curve with a shape model of the nucleus derived from visible data, and they estimate that the $R$-band albedo lies in the range 0.039-0.043. This value agrees with our best-fit value at the $2 \sigma$ level, and we attribute the discrepancy to a difference in the adopted values of the comet's absolute optical magnitude. Indeed, if we use the absolute magnitude $H_{R}=15.46$ derived by Lamy et al. (2008), our derivation of the comet's albedo decreases to $p_{R}=0.049 \pm 0.006$, which coincides within $1 \sigma$ of the Lamy et al. (2008) estimates. We 


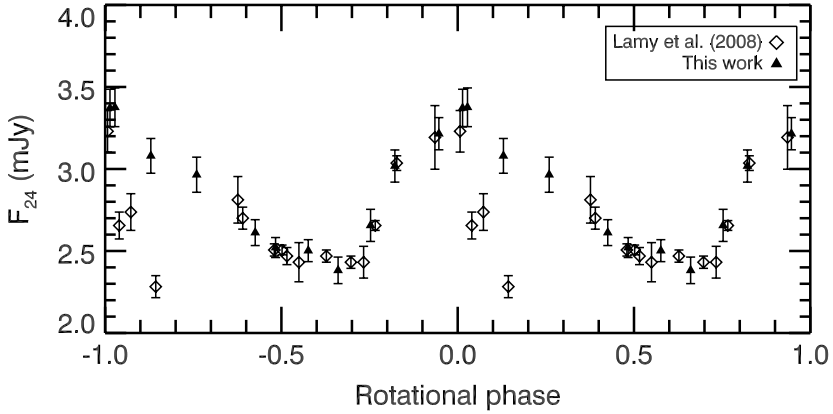

Figure 9. Spitzer MIPS $24 \mu \mathrm{m}$ (triangles) light curve (this work), and the pre-aphelion ( $r_{h}=4.5 \mathrm{AU}$ ) MIPS $24 \mu \mathrm{m}$ light curve from Lamy et al. (2008) (diamonds). We have phased the light curves with a $12.7047 \mathrm{hr}$ rotational period. The pre-aphelion light curve has been shifted in phase space and scaled by 0.78 to qualitatively match our post-aphelion data.

prefer to adopt the optical magnitude from Tubiana et al. (2008) since it was obtained when the comet was near aphelion, similar to our Spitzer observations.

Observations of 67P's visible light curve (Tubiana et al. 2008; Tubiana 2008) indicate that the overall variability profile is the same throughout the aphelion arc of the cometary orbit. Hence, we take the well-sampled $R$-band light curve of $67 \mathrm{P}$ from the 2006 May and August VLT data as a reference for comparison with the Spitzer measurements (Figure 10). Visual inspection of the light curves reveals that the maximum-to-minimum flux ratios (1.41 and 1.45) are similar. This result suggests that the variability at visible through mid-IR wavelengths is dominated by the effective radius of the nucleus as it rotates, rather than variations in the albedo.

Since it is not possible to do an exact phasing of the infrared and visible light curves, we applied arbitrary shifts to the visible light curve in order to force the maxima into agreement (Figure 10, top). The shifted light curves have significant differences at $0.1-0.6$ phases, and are systematically different at $0.4-0.6$ phases. We also attempted to minimize the point-topoint variations between the mid-IR and $R$-band light curves (Figure 10, bottom). The overall agreement has improved in this case, but significant differences still exist. The disagreements may indicate deviations in scattering and/or thermal behaviors of the surface, e.g., variations in albedo, surface roughness, or thermal conductivity. Or, there may be systematic errors in the photometry, which could explain a few rogue data points. For example, moving sources in the background are not accounted for with our background removal techniques, but may be faint and not apparent in the images. Such background sources have the greatest effect on the faintest parts of the light curve. It is interesting to note that Lamy et al. (2008) also had some difficulty finding a perfect agreement between their mid-IR light curve and a shape model derived using a light-curve inversion technique on optical photometry (Lamy et al. 2007). A further analysis of these discrepancies would require detailed shape, thermophysical, and scattered light models, better constrained phase shifts, and/or simultaneously obtained mid-IR and visible light curves. If we consider the overall agreements in peak-to-peak fluxes and the approximate agreement in lightcurve shapes, then we may place confidence in our nucleus albedo, color-temperature, and effective radius computed in Section 3.3.2.

\subsection{Nucleus Spectra}

In principle, our two spectra of the nucleus can provide an independent measurement of the nucleus effective temperature.

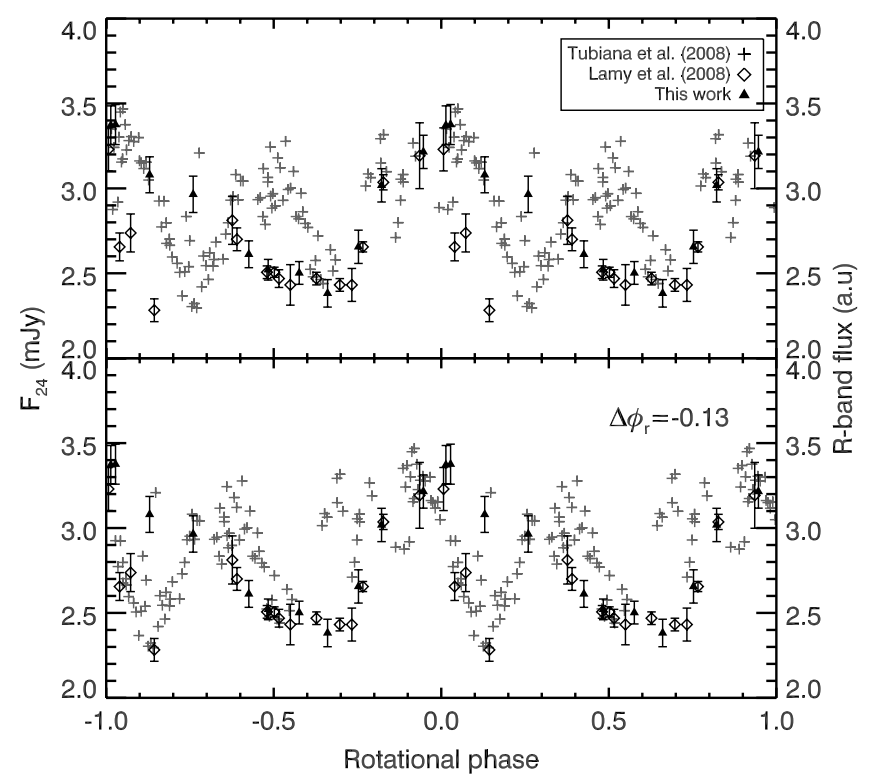

Figure 10. Same as Figure 9, but now the 2006 May and August $R$-band light curves (crosses) from Tubiana et al. (2008) have been added. The $R$-band light curve has been shifted to qualitatively match the Spitzer data. Top: the $R$-band data have been shifted to match the mid-IR light curve maximum. Bottom: the $R$-band data have been shifted by $\Delta \phi_{r}=-0.13$ phases so that the light curves better agree at 0.3-1.0 phases.

Both spectra (Figure 2) were taken during the post-aphelion $\operatorname{arc}\left(r_{h}=4.9\right.$ and $\left.4.3 \mathrm{AU}\right)$ along with our MIPS and IRAC observations. Using the NEATM, we fit two thermal models to each unbinned spectrum. In one model, the IR-beaming parameter is a free parameter, and in the other model, it is fixed at $\eta=0.68$ (Section 3.3.2). For both models the albedo is fixed at $p_{R}=0.054$. Our model results are presented in Figure 2, and the radii derived from the constant $\eta$ model fits are listed in Table 1. The absolute calibration error for the IRS instrument is approximately $10 \%$ (Spitzer Science Center 2008b), which is included in our radius uncertainties.

The IRS spectrum at $r_{h}=4.9 \mathrm{AU}$ does not significantly constrain the IR-beaming parameter $(\eta=1.3 \pm 0.4)$. Furthermore, when the IR-beaming parameter is allowed to vary, the best-fit radius increases to $3.0 \pm 0.5 \mathrm{~km}$ to compensate for the high best-fit $\eta$-value. Such a high $\eta$-value is unphysical given the constraints from our imaging data. A more meaningful IR-beaming parameter and effective radius is derived from the 4.3 AU spectrum: $R=1.99 \pm 0.21 \mathrm{~km}$ and $\eta=0.80 \pm 0.14$. This $\eta$-value agrees with the value of $0.68 \pm 0.06$ derived from the MIPS-IRAC light curve. Reduced $\chi^{2}$ values range from 0.6 to 0.8 for all model fits ( $\eta$-fixed and $\eta$-variable, $4.9 \mathrm{AU}$ and $4.3 \mathrm{AU})$.

\section{SUMMARY}

We observed comet 67P/Churyumov-Gerasimenko between 5.5 and $4.3 \mathrm{AU}$ from the Sun, post-aphelion, including a full light curve of the nucleus at 8 and $24 \mu \mathrm{m}$ at $4.8 \mathrm{AU}$. We employed the NEATM to derive a mean effective radius of $R=2.04 \pm 0.11 \mathrm{~km}$, and an average IR-beaming parameter of $\eta=0.68 \pm 0.06$. There is no evidence for variations of the surface temperature with rotational phase. The amplitude of the light curve constrains the primary-to-secondary axis ratio to $a / b \geqslant 1.41 \pm 0.07$. The mid-IR light curve is in good agreement with the MIPS $24 \mu \mathrm{m}$ light curve presented by Lamy et al. (2008), when both data sets are phased with a $12.7047 \mathrm{hr}$ rotation 
period (Tubiana et al. 2008), and when the Lamy et al. (2008) light curve is phase shifted to minimize point-to-point variations. Together, the composite mid-IR light curve roughly agrees with two aphelion $R$-band light curves measured by Tubiana et al. (2008) in 2006 May and August. Significant discrepancies exist between the two data sets, which may indicate surface variations of albedo, surface roughness, or thermal conductivity. However, the maximum-to-minimum flux ratios agree, suggesting that the nucleus variability is dominated by the shape of the nucleus as it rotates. Using the absolute magnitude $H_{R}=15.35 \pm 0.04$ (Tubiana et al. 2008), we estimate the $R$-band geometric albedo to be $p_{R}=0.054 \pm 0.007$.

The Spitzer spectrophotometric observations of the central point source are wholly commensurate with the thermal emission from an inactive nucleus. Therefore, we conclude that vigorous coma activity did not occur before $r_{h}=4.3 \mathrm{AU}$ in 2007. As the Rosetta spacecraft approaches the comet during the same in-bound path in 2014, we may expect to see images of an inactive or weakly active nucleus.

We also observed the dust trail and large dust grain tail (i.e., neck-line tail structure) surrounding the nucleus in $24 \mu \mathrm{m}$ images taken at 4.8 and 5.5 AU from the Sun. Our other images were not sensitive enough to detect the dust. Starting with the dust ejection parameters of Kelley et al. (2008) and Ishiguro (2008), and additionally imposing a maximum grain size of $6 \mathrm{~mm}\left(\beta>10^{-4}\right)$, simulations of the aphelion dust environment are consistent with our MIPS $24 \mu \mathrm{m}$ observations. Our maximum grain size agrees with the maximum grain size of $5 \mathrm{~mm}$ derived by Ishiguro (2008). The grain number density near the nucleus is $(1.33 \pm 0.03) \times 10^{-12} \mathrm{~m}^{-3}$, assuming that the dust is composed of millimeter-sized grains. The density corresponds to a Rosetta dust impact probability upper limit of $0.3 \%$ during the spacecraft's approach to the nucleus in 2014.

The authors acknowledge the Director of the Spitzer Science Center for providing Director's Discretionary Time, and appreciate the hard work of Vikki Meadows and the Observing Support Staff at the SSC for scheduling our rotationally phased light-curve observations, making possible the surface color-temperature measurements. This research made use of Tiny Tim/Spitzer, developed by John Krist for the Spitzer Science Center. This work is based in part on observations made with the Spitzer Space Telescope, which is operated by the Jet Propulsion Laboratory, California Institute of Technology under a contract with NASA. Support for this work was provided by NASA through contract 1289123 issued by JPL/Caltech to the University of Central Florida, and contracts 1263741, 1256406, and 1215746 issued by JPL/Caltech to the University of Minnesota. C.E.W. also acknowledges support from the National Science Foundation gran AST-0706980.

Facilities: Spitzer

\section{REFERENCES}

Agarwal, J., Boehnhardt, H., \& Gruen, E. 2007a, in Workshop on Dust in Planetary Systems, ESA SP-643, ed. H. Krueger \& A. Graps (Noordwijk: ESA), 51
Agarwal, J., Müller, M., \& Grün, E. 2007b, Space Sci. Rev., 128, 79

A'Hearn, M. F., et al. 2005, Science, 310, 258

Bowell, E., Hapke, B., Domingue, D., Lumme, K., Peltoniemi, J., \& Harris, A. W. 1989, in Asteroids II, ed. R. P. Binzel, T. Gehrels, \& M. S. Matthews (Tucson, AZ: Univ. Arizona Press), 524

Burns, J. A., Lamy, P. L., \& Soter, S. 1979, Icarus, 40, 1

Diolaiti, E., Bendinelli, O., Bonaccini, D., Close, L., Currie, D., \& Parmeggiani, G. 2000, A\&AS, 147, 335

Fazio, G. G., et al. 2004, ApJS, 154, 10

Fernández, Y. R., Campins, H., Kassis, M., Hergenrother, C. W., Binzel, R. P., Licandro, J., Hora, J. L., \& Adams, J. D. 2006, AJ, 132, 1354

Fernández, Y. R., et al. 2000, Icarus, 147, 145

Fulle, M., Barbieri, C., Cremonese, G., Rauer, H., Weiler, M., Milani, G., \& Ligustri, R. 2004, A\&A, 422, 357

Gehrz, R. D., et al. 2007, Rev. Sci. Instrum., 78, 1302

Glassmeier, K.-H., Boehnhardt, H., Koschny, D., Kührt, E., \& Richter, I. 2007, Space Sci. Rev., 128,

Green, S. F., et al. 2004, J. Geophys. Res. (Planets), 109, 12

Groussin, O., et al. 2007, Icarus, 187, 16

Hanner, M. S., Giese, R. H., Weiss, K., \& Zerull, R. 1981, A\&A, 104, 42

Harris, A. W. 1998, Icarus, 131, 291

Houck, J. R., et al. 2004, ApJS, 154, 18

Ishiguro, M. 2008, Icarus, 193, 96

Jewitt, D. 1991, in Comets in the Post-Halley Era, Vol. 1, ed. R. L. Newburn Jr. (Dordrect: Kluwer) 19

Jewitt, D. C., \& Meech, K. J. 1987, ApJ, 317, 992

Kadota, K., et al. 2008, Minor Planet Electron. Circ. L40

Kelley, M. S., Reach, W. T., \& Lien, D. J. 2008, Icarus, 193, 572

Kelley, M. S., et al. 2006, ApJ, 651, 1256

Kidger, M. R. 2003, A\&A, 408, 767

Kimura, H., \& Liu, C.-P. 1975, Acta Astron. Sin., 16, 138

Lamy, P. L., Toth, I., Davidsson, B. J. R., Groussin, O., Gutiérrez, P., Jorda, L. Kaasalainen, M., \& Lowry, S. C. 2007, Space Sci. Rev., 128, 23

Lamy, P. L., Toth, I., Fernandez, Y. R., \& Weaver, H. A. 2004, in Comets II, ed. M. Festou \& H. Keller, A. Weaver (Tucson, AZ: Univ. Arizona Press) 223

Lamy, P. L., Toth, I., Groussin, O., Jorda, L., Kelley, M. S., \& Stansberry, J. A. 2008, A\&A, 489, 777

Lamy, P. L., Toth, I., Weaver, H. A., Jorda, L., Kaasalainen, M., \& Gutiérrez, P. J. 2006, A\&A, 458, 669

Lowry, S. C., \& Fitzsimmons, A. 2001, A\&A, 365, 204

Lowry, S. C., Fitzsimmons, A., Cartwright, I. M., \& Williams, I. P. 1999, A\&A, 349,649

Lowry, S. C., Fitzsimmons, A., Jorda, L., Kaasalainen, M., Lamy, P., \& Toth, I. 2006, BAAS, 38, 492

Makovoz, D., \& Khan, I. 2005, in ASP Conf. Ser. 347, Astronomical Data Analysis Software and Systems XIV, ed. P. Shopbell, M. Britton, \& R. Ebert (San Francisco, CA: ASP) 81

McDonnell, J. A. M., et al. 1987, A\&A, 187, 719

McDonnell, J. A. M., et al. 1993, Nature, 362, 732

Mueller, B. E. A. 1992, in Proc. Asteroids, Comets, Meteors, 1991, ed. A. W. Harris \& E. Bowell (Houston, TX: Lunar and Planetary Inst.), 425

Reach, W. T., Vaubaillon, J., Kelley, M. S., Lisse, C. M., \& Sykes, M. V. 2009, Icarus, submitted

Rieke, G. H., et al. 2004, ApJS, 154, 25

Russell, H. N. 1916, ApJ, 43, 173

Snodgrass, C., Lowry, S. C., \& Fitzsimmons, A. 2008, MNRAS, 385, 737

Southworth, R. B. 1963, AJ, 68, 293

Spitzer Science Center 2006, in Infrared Array Camera Data Handbook, (Pasadena, CA: SSC) http://ssc.spitzer.caltech.edu/irac/dh/

Spitzer Science Center 2008a, in Multiband Imaging Photometer for Spitzer (MIPS) Data Handbook, (Pasadena, CA: SSC) http://ssc.spitzer.caltech.edu/ $\mathrm{mips} / \mathrm{dh} /$

Spitzer Science Center 2008b, in Infrared Spectrograph Data Handbook, (Pasadena: SSC) http://ssc.spitzer.caltech.edu/irs/dh/

Sykes, M. V., \& Walker, R. G. 1992, Icarus, 95, 180

Tubiana, C. 2008, PhD thesis, Max Planck Institute for Solar System Research Tubiana, C., Barrera, L., Drahus, M., \& Boehnhardt, H. 2008, A\&A, 490, 377 Werner, M. W., et al. 2004, ApJS, 154, 1

Wooden, D. W., Kelley, M. S., Woodward, C. E., Harker, D. E., Boehnhardt, H., Tubiana, C., \& Prialnik, D. 2008, in LPI Contributions 1405, 8380 